\title{
Processamento e caracterização de filtros cerâmicos fibrosos
}

\section{(Processing and characterization of fibrous ceramic filters)}

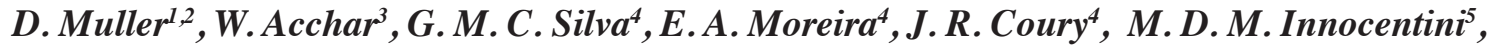 \\ D. Hotzal, ${ }^{1,2}$ C.R.Rambo ${ }^{1,2 *}$ \\ ${ }^{1}$ Programa de Pós-Graduação em Ciência e Engenharia de Materiais - PGMAT, ${ }^{2}$ Departamento de Engenharia \\ Química e Engenharia de Alimentos - EQA, Universidade Federal de Santa Catarina - UFSC, \\ Florianópolis, SC 88040-900 \\ ${ }^{3}$ Universidade Federal do Rio Grande do Norte - UFRN, 59072-970 Natal, RN \\ ${ }^{4}$ Departamento de Engenharia Química (DEQ), Universidade Federal de S. Carlos - UFSCar, \\ S. Carlos, SP 13565-905 \\ ${ }^{5}$ Curso de Engenharia Química, Universidade de Ribeirão Preto - UNAERP, Ribeirão Preto, SP 14096-900
}

\begin{abstract}
Resumo
Neste trabalho, filtros com estrutura fibrosa foram produzidos utilizando-se matéria-prima de baixo custo, disponível comercialmente e caracterizados para aplicações em filtração de aerossóis. Mantas refratárias sílico-aluminosas comerciais foram prensadas uniaxialmente, utilizando-se $10 \%$ em massa de acetato de polivinila como ligante. Nesta etapa, as amostras foram submetidas à compactação com diferentes pressões para obtenção de diferentes porosidades. Após a prensagem, as amostras foram submetidas a um tratamento térmico a $500{ }^{\circ} \mathrm{C}$ durante $1 \mathrm{~h}$ para a degradação do polímero. Subseqüentemente, as amostras foram sinterizadas a $1200{ }^{\circ} \mathrm{C}$ durante $1 \mathrm{~h}$, resultando em uma estrutura fibrilar porosa, composta por mulita $\left(3 \mathrm{SiO}_{2} .2 \mathrm{Al}_{2} \mathrm{O}_{3}\right)$, com porosidade na faixa de 50 a 75\%. A morfologia dos filtros fibrosos foi caracterizada através de microscopia eletrônica de varredura, que revelou uma estrutura tridimensional porosa de fibras interconectadas. A resistência mecânica foi avaliada através de ensaios de compressão e de flexão em quatro pontos. Ensaios de permeabilidade e eficiência de coleta de partículas com diferentes diâmetros foram efetuados e os resultados comparados a filtros comerciais. Os valores obtidos para a permeabilidade e eficiência de coleta estão na ordem de grandeza esperada para filtros de gases, apresentando assim grande potencial para aplicações industriais.
\end{abstract}

Palavras-chave: filtros cerâmicos fibrosos, propriedades mecânicas, permeabilidade, filtração de aerossóis.

\begin{abstract}
Filters with fibrous structure were produced from low cost, commercially available raw materials and characterized for aerosol filtration. Refractory aluminosilicate fibers were uniaxially pressed with polyvinyl acetate as binder. Different pressures were applied, which yielded samples with different porosities. After pressing, the samples were heat treated at $500{ }^{\circ} \mathrm{C}$ during $1 \mathrm{~h}$ in air for debinding with subsequent sintering at $1200^{\circ} \mathrm{C}$ for $1 \mathrm{~h}$, which resulted in porous fibrous structures composed mainly by mullite $\left(3 \mathrm{SiO}_{2} .2 \mathrm{Al}_{2} \mathrm{O}_{3}\right)$ with porosity in the range of 50\%-75\%. The microstructure of the fibrous filters was evaluated by scanning electron microscopy, which revealed a porous morphology comprised by three-dimensional interconnected fibers. Mechanical strength was measured by compressive and 4-point bending tests. Permeability and particle collection efficiency were evaluated and compared with commercially available filters. The permeability and collection efficiency values are in the order of magnitude of cellular supports for aerosol filters and, therefore, suitable for several technological applications.
\end{abstract}

Keywords: fibrous ceramic filters, mechanical properties, permeability, aerosol filtration.

\section{INTRODUÇÃO}

Filtros cerâmicos têm despertado grande interesse tecnológico por resistirem a temperaturas superiores a $1000{ }^{\circ} \mathrm{C}$, apresentando alta eficiência de filtração e baixo custo de fabricação e manutenção. Entre várias aplicações dos filtros cerâmicos destacam-se queima de resíduos, refinamento de metais, combustão de óleo diesel e filtração de gases quentes $[1,2]$. Uma característica da operação unitária de filtração de partículas sólidas presentes em um fluido é a diversidade de equipamentos disponíveis. Tal diversidade por sua vez tem sido conseqüência da multiplicidade dos problemas de filtração, nas quais partículas, em suspensões de alta ou baixa concentração, devem ser separadas dos gases, que podem ser corrosivos e, em alguns casos, explosivos [3]. De acordo com suas estruturas e constituintes, os filtros cerâmicos podem ser divididos em duas categorias principais: filtros granulares e fibrosos. Os principais candidatos cerâmicos para o uso de filtros são cordierita, mulita, alumina e carbeto 
de silício. As exigências para estes filtros cerâmicos, cuja função principal é remover as partículas entre $0,2 \mathrm{e}$ $10 \mu \mathrm{m}$, são: permeabilidade elevada, porosidade de 50 a $80 \%$, resistência à erosão e resistência ao choque térmico. De acordo com os materiais indicados para cada tipo de aplicação, o filtro depende criticamente da microestrutura e das propriedades mecânicas e térmicas associadas a esses materiais [4]. A eficiência dos meios fibrosos para remover os materiais particulados depende de diversos fatores, incluindo diâmetro e orientação das fibras, velocidade e carga de particulados no fluido [5]. A coleta das partículas presentes em um fluxo de gás ocorre através de vários mecanismos. No caso de filtração de ar em meios fibrosos, o mecanismo difusional é mais eficiente para partículas menores que $0,2 \mu \mathrm{m}$. Os mecanismos inercial, gravitacional e de interceptação direta são mais eficientes na filtração de partículas maiores que $2 \mu \mathrm{m}$. Desta maneira, a eficiência total de coleta das partículas é admitida como sendo uma combinação de todos os mecanismos [6]. Idealmente, o filtro cerâmico deve remover o máximo de impurezas com a mínima resistência ao fluido de arraste [7]. Permeabilidade, eficiência de coleta e resistência mecânica são parâmetros que devem ser otimizados de acordo com o processo de interesse. As interações complexas e dinâmicas que ocorrem entre as partículas e a superfície do filtro, no entanto, tornam o comportamento do filtro cerâmico especialmente dependente das condições de operação e do tipo de aerossol envolvido [8].

Uma relação permeabilidade/resistência mecânica adequada pode ser obtida através da combinação ideal do tamanho do poro e da porosidade [9]. Nesse caso, a permeabilidade e a resistência mecânica, tanto de estruturas celulares como de estruturas fibrosas, apresentamse de maneiras distintas. Poros grandes favorecem a permeabilidade, mas reduzem a eficiência de coleta das partículas e diminuem a resistência mecânica. Por outro lado, poros pequenos aumentam a eficiência de coleta, contribuem para o aumento da resistência mecânica, mas promovem o aumento da queda de pressão através do filtro $[10,11]$. A permeabilidade é um parâmetro-chave quando o material é projetado com a finalidade de filtração. A permeabilidade de um elemento poroso pode ser descrita pela equação de Forchheimer (equação A), que indica uma tendência parabólica da queda de pressão com a velocidade do fluido superficial $\left(v_{\mathrm{s}}\right)$ [12]. A equação A é composta por um termo linear, que expressa os efeitos do atrito (regime viscoso) e um termo quadrático, que descreve a contribuição dos efeitos cinéticos (inércia e turbulência) sobre a queda de pressão:

$$
\frac{\mathrm{P}_{\mathrm{e}}^{2}-\mathrm{P}_{\mathrm{s}}^{2}}{2 \mathrm{PL}}=\frac{\mu}{\mathrm{k}_{1}} \mathrm{v}_{\mathrm{s}}+\frac{\rho}{\mathrm{k}_{2}} \mathrm{v}_{\mathrm{s}}^{2}
$$

na qual $\mathrm{P}_{\mathrm{e}}$ é a pressão absoluta do fluido na entrada da amostra, $\mathrm{P}_{\mathrm{s}}$ é a pressão absoluta na saída, $\mathrm{P}$ é a pressão na qual a vazão é medida e as propriedades físicas são calculadas (neste trabalho, $\mathrm{P}=\mathrm{P}_{\mathrm{e}}$ ), $\mathrm{L}$ é a espessura do filtro, e são, respectivamente, a viscosidade e a densidade do fluido (neste trabalho, obtidas para temperatura de $25^{\circ} \mathrm{C}$ e pressão atmosférica de $\left.1,013 \times 10^{5} \mathrm{~Pa}\right), \mathrm{k}_{1}$ e $\mathrm{k}_{2}$ são as constantes de permeabilidade Darciana e não-Darciana, respectivamente. Ambas as permeabilidades dependem exclusivamente das estruturas e dos poros [10].

Quando os efeitos cinéticos são negligenciados, a equação A reduz-se à lei de Darcy, indicando somente a dependência linear da velocidade do gás em relação ao gradiente de pressão. Filtros cerâmicos comerciais com porosidades entre 70 e $90 \%$ apresentam permeabilidade Darciana $\mathrm{k}_{1}$ tipicamente na faixa de $10^{-12}$ a $10^{-8} \mathrm{~m}^{2}$ e resistência à compressão que varia de 0,5 a $2,0 \mathrm{MPa}[2,13,14]$.

Este trabalho teve como objetivo desenvolver filtros cerâmicos fibrosos usando matéria-prima comercialmente disponível e economicamente viável. Os filtros produzidos foram caracterizados nos termos de suas propriedades mecânicas, bem como sua permeabilidade e eficiência de filtração.

\section{PROCEDIMENTO EXPERIMENTAL}

\section{Matérias-primas e processamento}

Os filtros cerâmicos com estrutura fibrosa foram desenvolvidos a partir de mantas refratárias silico-aluminosas comerciais com densidade de $2840 \mathrm{~kg} / \mathrm{m}^{3}\left(50 \%\right.$ de $\mathrm{SiO}_{2}, 49 \%$ de $\mathrm{Al}_{2} \mathrm{O}_{3}$, além de conterem traços inorgânicos). As fibras foram prensadas uniaxialmente com o auxílio de ligante à base de acetato de polivinila (PVA) em uma matriz cilíndrica de $50 \mathrm{~mm}$ de diâmetro e $10 \mathrm{~mm}$ de espessura. Diferentes porosidades foram obtidas submetendo as amostras a pressões entre 17 e $41 \mathrm{MPa}$. Depois de prensadas, as amostras foram tratadas termicamente para degradação e liberação do polímero a $500{ }^{\circ} \mathrm{C}$ por $1 \mathrm{~h}$ com taxa de aquecimento de 10 ${ }^{\circ} \mathrm{C} / \mathrm{min}$. Após essa etapa, as amostras foram sinterizadas a $1200{ }^{\circ} \mathrm{C}$ por $1 \mathrm{~h}$, que resultou em estruturas fibrosas com porosidades entre 50 e $75 \%$.

\section{Caracterização física e microestrutural}

Para a determinação das fases cristalinas presentes na matéria-prima fibrosa, bem como das fases cristalinas formadas após o tratamento térmico, foi utilizada a difratometria de raios X (DRX, Philips PW 3710), utilizando radiação Cuk $\alpha$. Para identificação das fases cristalinas formadas, utilizou-se o banco de dados JCPDS. A densidade das fibras $\left(\rho_{s}\right)$ foi determinada por picnometria utilizando gás hélio (Multi Pycnometer, Quantachrome MVP-4DC) a $25{ }^{\circ} \mathrm{C}$ a partir das amostras prensadas e sinterizadas. A porosidade das amostras $(\varepsilon)$ foi calculada pela relação entre densidade picnométrica e densidade geométrica ou aparente $\left(\varrho_{0}\right)$ de acordo com a equação B:

$$
\varepsilon=\left[100 x\left(1-\rho_{0} / \rho_{s}\right)\right]
$$


A morfologia e a microestrutura dos filtros fibrosos foram avaliadas por microscopia eletrônica de varredura (MEV, XL-30, Philips) juntamente com análise por espectrometria de energia dispersiva (EDX).

Os ensaios de resistência à compressão foram realizados a $25^{\circ} \mathrm{C}$ em uma máquina universal de ensaios (Emic DL 2000). A velocidade de carregamento aplicada foi $1 \mathrm{~mm} / \mathrm{min}$. Foram utilizados pelo menos 10 corpos-de-prova com dimensões nominais de $13 \times 10 \times 7 \mathrm{~mm}^{3}$. As superfícies dos corpos-deprova ortogonais à aplicação da carga de compressão foram cobertas com uma borracha flexível, para eliminar efeitos de carregamento localizado, devido à topografia da superfície das amostras. Para os ensaios de resistência à flexão em 4 pontos a $25{ }^{\circ} \mathrm{C}$ foram utilizados pelo menos 10 corpos-de-prova com dimensões de $60 \times 20 \times 7 \mathrm{~mm}^{3}$. Estes ensaios foram realizados usando uma máquina de ensaio de materiais (Z2.5/TS1S-2004, Zwick/Roell). A velocidade de carregamento foi de $1 \mathrm{~mm} / \mathrm{min}$. Os ensaios foram feitos com carga perpendicular à orientação da fibra. As resistências máximas sob compressão e flexão foram determinadas através da medida do primeiro pico de tensão na região de deformação elástica nas respectivas curvas tensão-deformação.

\section{Permeabilidade e eficiência de coleta}

A permeabilidade dos meios fibrosos foi medida em temperatura ambiente em um permeâmetro dividido em duas seções cilíndricas, entre as quais está acoplado um portaamostras cilíndrico. Os filtros foram dispostos no portaamostras entre as seções cilíndricas e vedados de forma a evitar escoamento radial. O diâmetro efetivo de escoamento foi de $3 \mathrm{~cm}$. A vazão de ar foi suprida por um soprador de 7,5 HP e medida em um rotâmetro. A queda de pressão através do filtro foi medida com um micromanômetro (FCO14, Furness Control). Os ensaios consistiram na passagem de ar através do elemento filtrante e medição da queda de pressão resultante. Um mínimo de 10 pares de vazão - queda de pressão foram obtidos nos ensaios. Para cada amostra, foram feitas tréplicas das curvas experimentais.

A filtração foi realizada verticalmente em sentido ascendente no mesmo equipamento utilizado nos ensaios de permeabilidade. As partículas na entrada e na saída do filtro foram quantificadas por meio de contador de partículas (Haic/Royco Modelo 5230), em dez faixas de tamanho entre 0,5 e $20 \mu \mathrm{m}$. Por meio da densidade do material particulado e do número de partículas na entrada e na saída do filtro, foi possível obter as massas de partículas ( $\mathrm{M}_{\text {entrada }}$ e $\left.\mathrm{M}_{\text {saida }}\right)$, por faixa de tamanho. O material particulado utilizado neste experimento foi um concentrado fosfático com densidade de $2970 \mathrm{~kg} / \mathrm{m}^{3}$. A eficiência de coleta experimental, por faixa granulométrica $\left(\mathrm{E}_{\mathrm{i}}\right)$, foi determinada pela equação $\mathrm{C}$ :

$$
\mathrm{E}_{\mathrm{i}}=\frac{\mathrm{N}_{\mathrm{e}, \mathrm{i}}-\mathrm{N}_{\mathrm{S}, \mathrm{i}}}{\mathrm{N}_{\mathrm{e}, \mathrm{i}}} \cdot 100 \%
$$

na qual $\mathrm{N}_{\mathrm{e}, \mathrm{i}}$ é o número de partículas com diâmetro $\mathrm{d}_{\mathrm{pi}}$ na entrada do filtro, $\mathrm{N}_{\mathrm{s}, \mathrm{i}}$ é o número de partículas com diâmetro $\mathrm{d}_{\mathrm{pi}}$ na saída do filtro, i é a faixa de tamanho analisada. O ensaio foi realizado em duas etapas, a fim de se obter o número de partículas na saída do filtro e o número total de partículas de entrada no filtro, por faixa de tamanho.

\section{RESULTADOS E DISCUSSÃO}

\section{Fases e microestrutura}

A Fig. 1 apresenta os difratogramas de raios $\mathrm{X}$ das fibras como recebidas e após tratamento térmico a $1200{ }^{\circ} \mathrm{C}$ durante $1 \mathrm{~h}$. Observa-se que a amostra de fibras de aluminossilicato como recebida apresenta-se amorfa. Com o aumento da temperatura, a $1200{ }^{\circ} \mathrm{C}$ ocorreu a formação de mulita $\left(3 \mathrm{SiO}_{2} .2 \mathrm{Al}_{2} \mathrm{O}_{3}\right)$, como fase predominante, apresentando fases residuais de sílica amorfa (halo em torno de $2 \theta=20^{\circ}$ ).

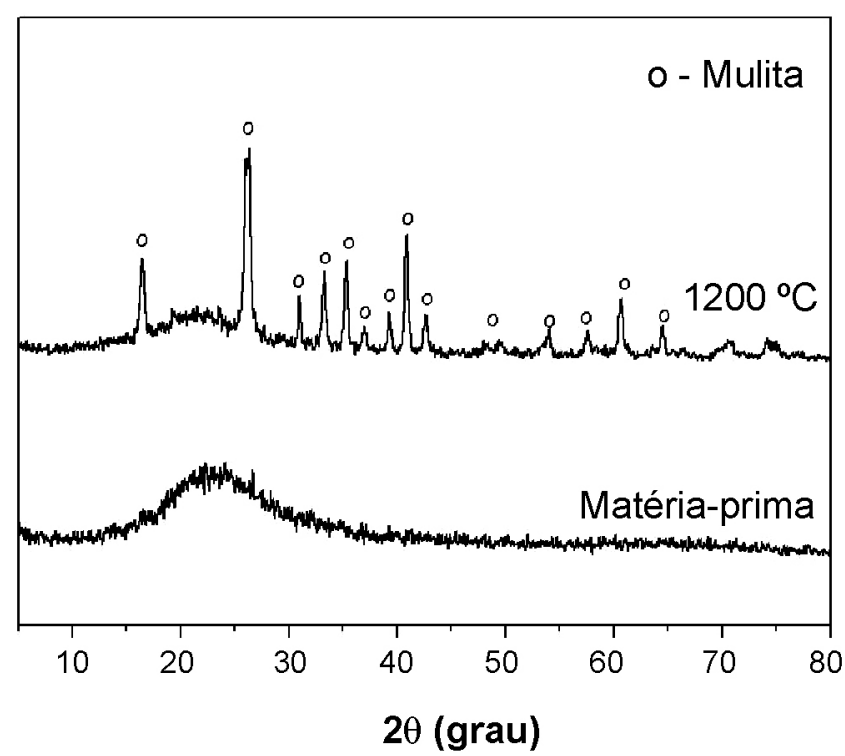

Figura 1: Difratogramas de raios $\mathrm{X}$ de amostras de fibras como recebidas (manta refratária) e após tratamento térmico a $1200{ }^{\circ} \mathrm{C}$ durante $1 \mathrm{~h}$.

[Figure 1: X-ray diffraction patterns of the fibers: as-received and after thermal treatment at $1200{ }^{\circ} \mathrm{C}$ for $\left.1 \mathrm{~h}.\right]$

A Fig. 2 apresenta micrografias obtidas por MEV (Figs. 2a-d) e análise química por EDX (Figs. 2e-f) do material fibroso como recebido (manta refratária com $96 \%$ de porosidade) e dos filtros fibrosos sinterizados por $60 \mathrm{~min}$ a $1200{ }^{\circ} \mathrm{C}$ com diferentes porosidades. A Fig. $2 \mathrm{a}$ apresenta a micrografia do material fibroso como recebido, mostrando uma rede de fibras longas interconectadas, arranjadas aleatoriamente. $\mathrm{O}$ diâmetro da fibra está distribuído na faixa entre $1 \mu$ e $100 \mu \mathrm{m}$, como mostrado em detalhe na Fig. 2a. Com a diminuição da porosidade, a superfície da amostra torna-se mais densa. Isto pode ser observado na amostra com $73 \%$ de porosidade na Fig. 2b. Em amostras com menores porosidades, estas diferenças morfológicas não são observadas com clareza (Figs. 2c-d). 

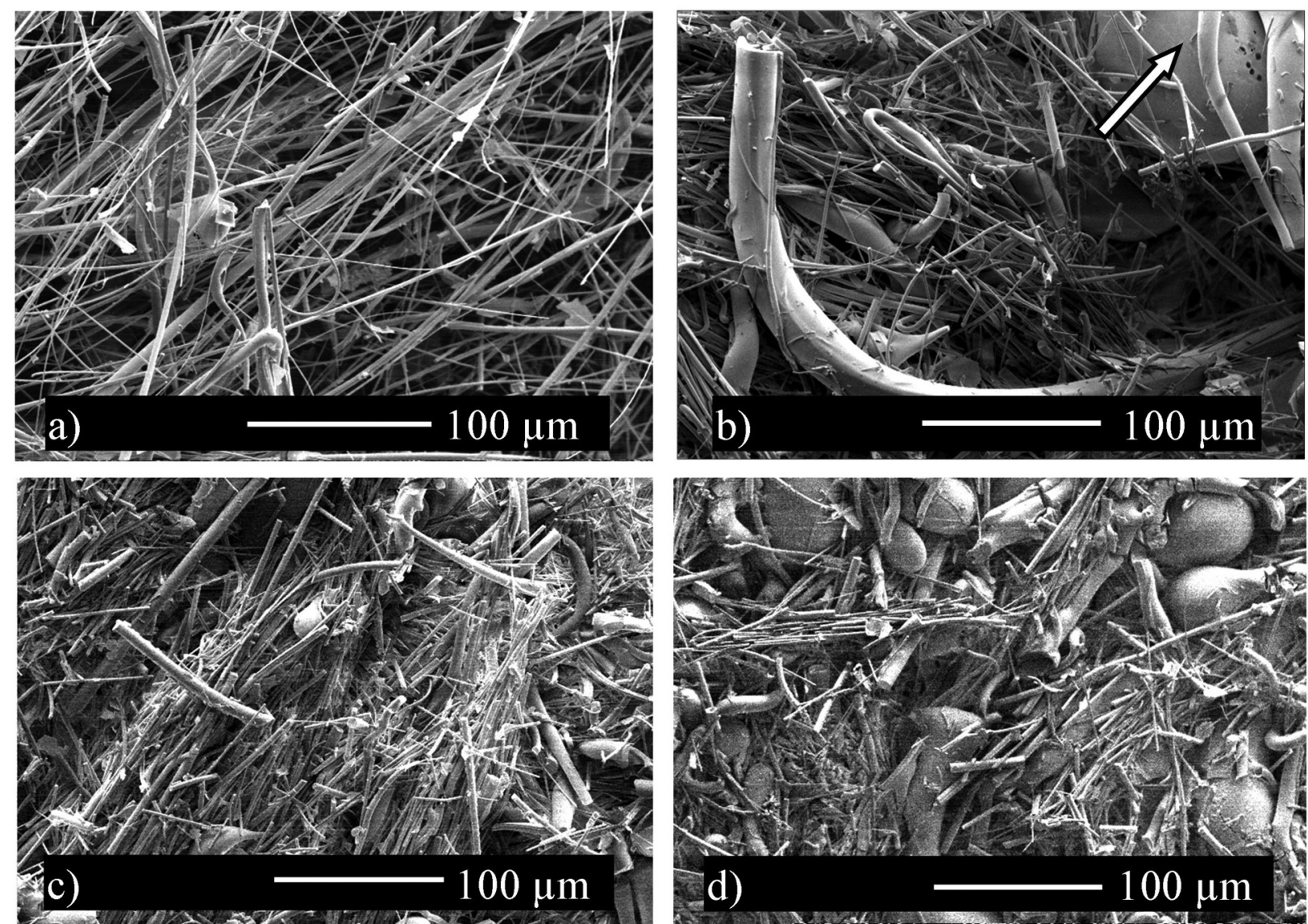

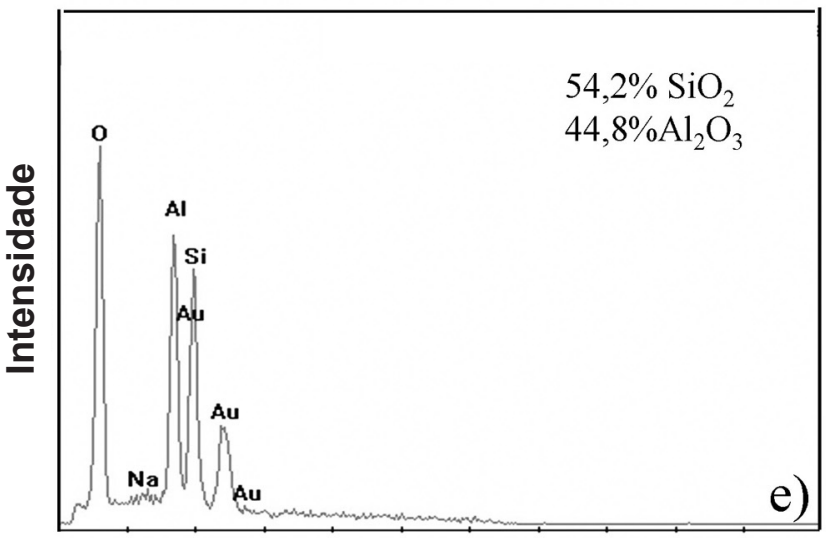

Energia (keV)

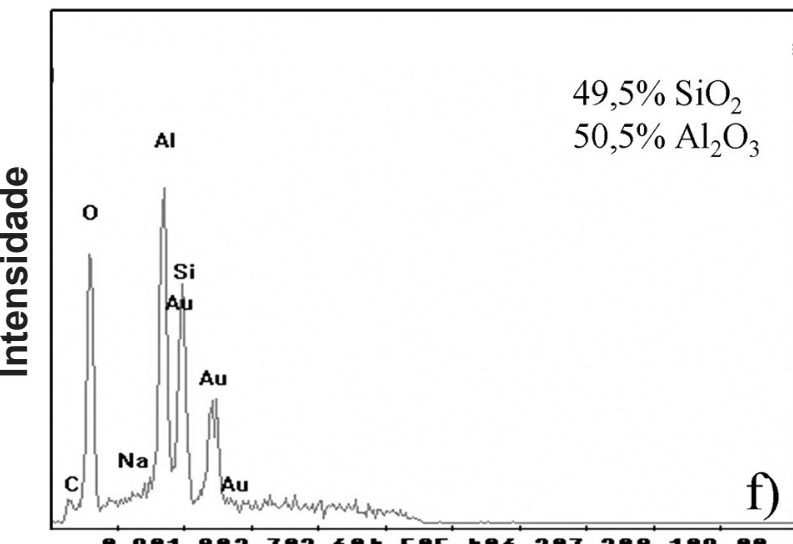

Energia (keV)

Figura 2: Micrografias obtidas por MEV e análise de EDX dos materiais fibrosos com diferentes porosidades: a) fibras como recebidas (95\%); b) 73\%; c) 65\%; d) 50\%; e) EDX da partícula evidenciada pela seta na Fig. 2b; f) EDX da superfície de uma fibra.

[Figure 2: SEM micrographs of the fibrous materials with different porosities: a) as-received (95\%), b) 73\%; c) 65\%; d) 50\%.e) EDX of the particle indicated by the arrow in Fig. $2 b$; $f$ ) EDX of the surface of a fiber.]

Após o tratamento térmico, os filtros são compostos de extensas fibras com diferentes diâmetros. Durante o tratamento térmico ocorreu a formação de uma fase líquida de $\mathrm{SiO}_{2}$ com subseqüente encurtamento das fibras, originando partículas arredondadas (globulares), distribuídas entre o material fibroso (seta na Fig. 2b). Esse comportamento é característico da síntese de fibras de mulita, a partir da devitrificação de fibras aluminossilicosas amorfas [15]. Esse tipo de morfologia tem início nas pontas das fibras e está relacionado à diminuição da área de superfície por unidade de volume, que leva assim a um formato esférico. A análise química pontual por EDX revelou um teor maior de $\mathrm{SiO}_{2}$ na superfície dessas partículas (Fig. 2e), em comparação com a superfície das fibras (Fig. 2f), o que indica que a fase líquida formou-se na superfície. A presença desse tipo de morfologia aliada à ampla distribuição do tamanho das 
fibras pode contribuir para acentuar a eficiência de coleta de partículas, visto que diferentes mecanismos de filtração atuam dependendo do tamanho de partícula a ser coletada $[2,4]$.

\section{Propriedades mecânicas}

Curvas típicas força-deformação para filtros cerâmicos fibrosos com diferentes porosidades para ensaios mecânicos sob compressão são mostradas na Fig. 3 .

De acordo com o modelo de Gibson-Ashby (GA), que descreve o comportamento mecânico de estruturas celulares, a falha de materiais cerâmicos celulares, bem como em malhas de fibras cerâmicas ocorre por fratura frágil [16]. Na Fig. 3 observam-se três estágios principais na curva tensão-deformação de cerâmicas com estrutura celular, sob compressão: 1) regime linear elástico, correspondendo à flexão das fibras. Nesse estágio, observa-se que a amostra com 50\% de porosidade apresentou uma carga máxima de 86 $\mathrm{N}$ e a amostra com $65 \%$ de porosidade alcançou uma carga máxima de $27 \mathrm{~N}$; 2) patamar de tensão, correspondendo à fratura progressiva das fibras, observado especialmente nas amostras com 50 e $65 \%$ de porosidade; e 3) densificação, correspondente à extinção dos espaços vazios por todo o material com simultânea compactação das fibras umas sobre as outras [16].

A Fig. 4 mostra a resistência máxima sob compressão em função da densidade relativa $\left(\varrho_{\text {rel }}=\varrho_{o} / \varrho_{s}\right)$ dos filtros cerâmicos fibrosos. A resistência máxima variou entre $0,3 \mathrm{e}$ 1,5 MPa para porosidades entre 78 e $57 \%$, respectivamente. A resistência à compressão diminui com o aumento da densidade relativa, como esperado para materiais porosos [16]. Foi observado, ainda, que valores muito próximos de densidade relativa apresentaram valores em uma ampla faixa de resistência, o que provavelmente está relacionado com variações locais na distribuição de tamanho das fibras,

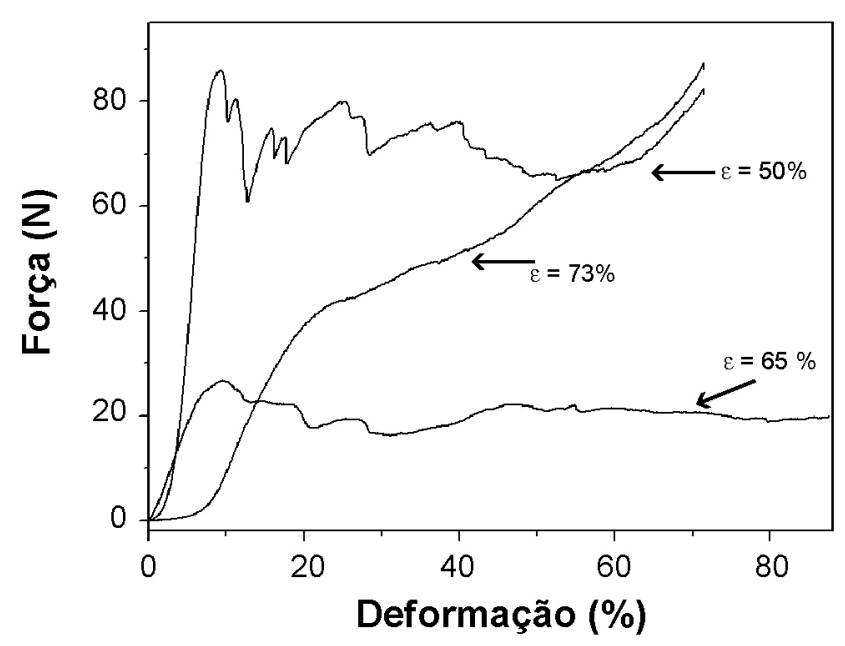

Figura 3: Curvas tensão-deformação dos filtros cerâmicos fibrosos com diferentes porosidades, sob compressão.

[Figure 3: Compressive stress-strain curves of the ceramic fibrous filters with different porosities.]

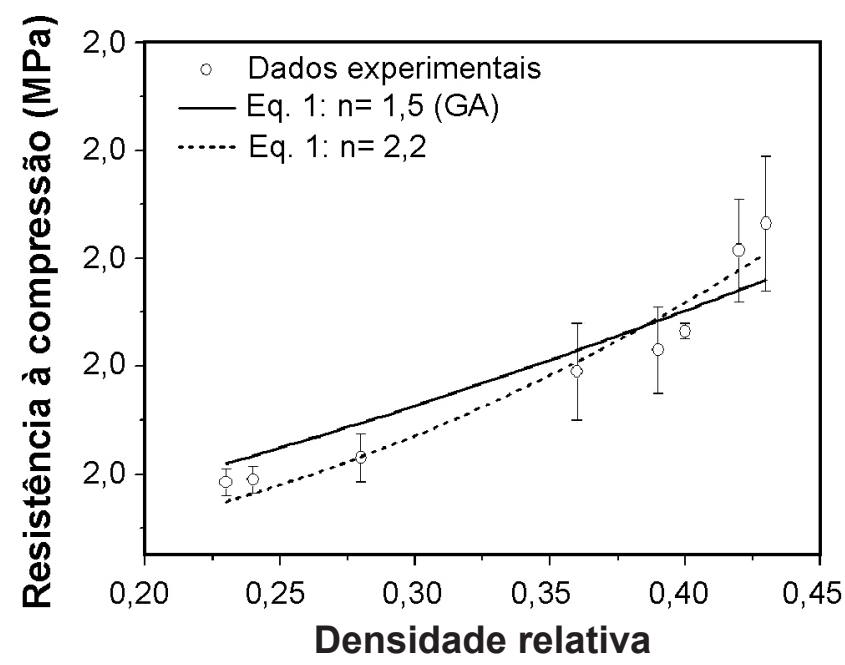

Figura 4: Resistência à compressão em função da densidade relativa das cerâmicas fibrosas.

[Figure 4: Compressive strength as a function of the relative density of the fibrous ceramics.]

provenientes do processo de conformação. Isso acarretou desvios substanciais na média de resistência à compressão para uma mesma porosidade, como pode ser observado na Fig. 4.

A relação genérica para a dependência da resistência mecânica com a porosidade é dada pela Equação D [17]:

$$
\frac{\sigma_{0}}{\sigma_{s}}=C_{1}\left(\frac{\rho_{0}}{\rho_{s}}\right)^{n}
$$

na qual $\sigma_{\mathrm{o}}$ é a resistência do material poroso, $\sigma_{\mathrm{s}}$ é resistência da fração sólida, $\rho_{\mathrm{o}} / \rho_{\mathrm{s}}$ é a densidade relativa, $\mathrm{C}_{1}$ é uma constante relacionada com a forma da célula e o expoente $\mathrm{n}$ é uma constante que depende da tortuosidade, como uma medida de desordem no material e está relacionado com a distribuição e geometria da célula.

Para $\mathrm{n}=1,5$, a equação D se reduz ao modelo de GibsonAshby para cerâmicas celulares com células abertas, baseado no colapso catastrófico das células sob determinado valor de tensão crítica. Nesse caso, $\mathrm{n}$ é derivado de considerações geométricas para o cálculo de $\sigma_{0}$, utilizandose a teoria de deformações de vigas $[16,18]$. Ajustando os dados experimentais pela equação $\mathrm{D}$ e fixando o valor de n como 1,5 (modelo de Gibson-Ashby, curva sólida na Fig. 4), pode ser observado um desvio maior para baixos valores de densidade relativa. Uma melhor correlação foi obtida quando os dados experimentais foram ajustados com a mesma equação, porém sem fixar o valor de $n$. Neste caso, foi encontrado para a estrutura fibrosa $\mathrm{n}$ igual a 2,2 (curva pontilhada na Fig. 4). Para $n$ igual a 1,5, o valor de $\mathrm{C}_{1}$ é 0,007 (coeficiente de correlação $=0,88$ ) e, para $\mathrm{n}$ igual a 2,2, o valor de $\mathrm{C}_{1}$ é 0,015 (coeficiente de correlação $=0,95$ ). A diferença no valor de $\mathrm{n}$ pode ser explicada pelo fato do modelo de Gibson-Ashby se basear no colapso catastrófico de células homogêneas sem defeitos em seus filamentos sólidos. Como o material é composto por uma 


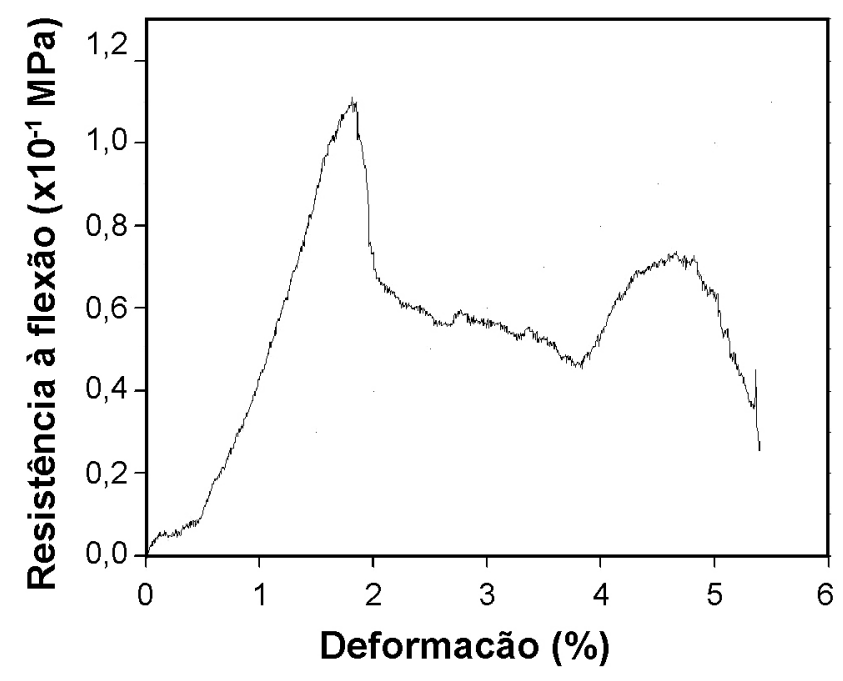

Figura 5: Curva tensão-deformação de um filtro com $70 \%$ de porosidade sob teste de flexão a 4-pontos.

[Figure 5: Four-point flexural stress-strain curve of a filter with $70 \%$ porosity.]

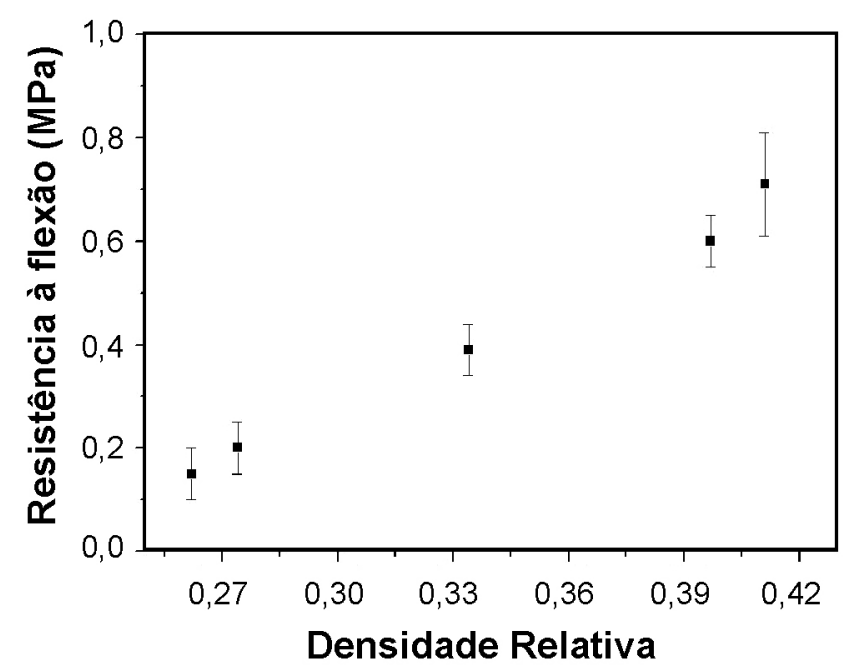

Figura 6: Resistência à flexão em função da densidade relativa dos filtros cerâmicos fibrosos.

[Figure 6: Bending strength as a function of the relative density of the fibrous ceramics.]

rede de fibras heterogêneas, com ampla distribuição de diâmetros, desvios do modelo são naturalmente esperados. Ainda, o valor de $\mathrm{n}$ depende do modelo micromecânico utilizado para descrever a estrutura celular/fibrosa, dos defeitos microestruturais existentes nos filamentos sólidos e também do mecanismo de transferência de carga entre os constituintes do material [19]. Adicionalmente, valores mais baixos de $\sigma_{\mathrm{o}}$ podem ser atribuídos a uma desordem das células/poros (não-homogeneidade de tamanho e morfologia), gerando valores mais elevados de $n$.

$\mathrm{Na}$ Fig. 5, que representa uma curva típica de resistência à flexão em função da deformação, podem ser observados os três regimes, analogamente aos ensaios de resistência à compressão: 1) regime linear elástico, em que foi determinada uma resistência máxima de 0,15 $\mathrm{MPa}$, nesse caso relacionada ao envergamento elástico das fibras; 2) patamar de tensão, que corresponde ao colapso progressivo das fibras; e 3) densificação, correspondente ao intervalo de deformação observado de $0,5 \%$, seguido pela ruptura da amostra.

A Fig. 6 apresenta a resistência à flexão dos filtros cerâmicos fibrosos em função da densidade relativa. Sob flexão, as cerâmicas fibrosas apresentam comportamento semelhante ao daquele sob compressão, isto é, a resistência mecânica aumenta com a diminuição da porosidade. Ainda que os filtros fibrosos apresentem uma resistência mecânica relativamente baixa, suas características mecânicas são adequadas para o manuseio dos filtros em condições reais de operação, como troca ou montagem.

\section{Permeabilidade}

A Fig. 7 mostra a queda de pressão em função da velocidade do ar para os filtros fibrosos testados. Podese observar que ocorreu um aumento não linear na queda de pressão com o aumento da velocidade, mostrando que houve influência de forças inerciais e viscosas sobre a queda de pressão. A queda de pressão é maior para amostras com baixa porosidade. Para altas porosidades, contudo os efeitos inerciais tornam-se mais evidentes, isto é, maior a perda de energia (pressão) associada à turbulência e à mudança de trajetória no interior dos poros. Deste modo, o termo parabólico da equação de Forchheimer torna-se mais importante do que o termo linear. Nesta região, qualquer alteração de velocidade potencializa a variação na queda de pressão, o que é indesejado para o processo de filtração.

A Fig. 8 mostra a influência da porosidade sobre as constantes de permeabilidade Darciana e não-Darciana, $\mathrm{k}_{1}$ e $\mathrm{k}_{2}$, obtidas através do ajuste dos dados experimentais apresentados na Fig. 7, utilizando-se a equação A. As

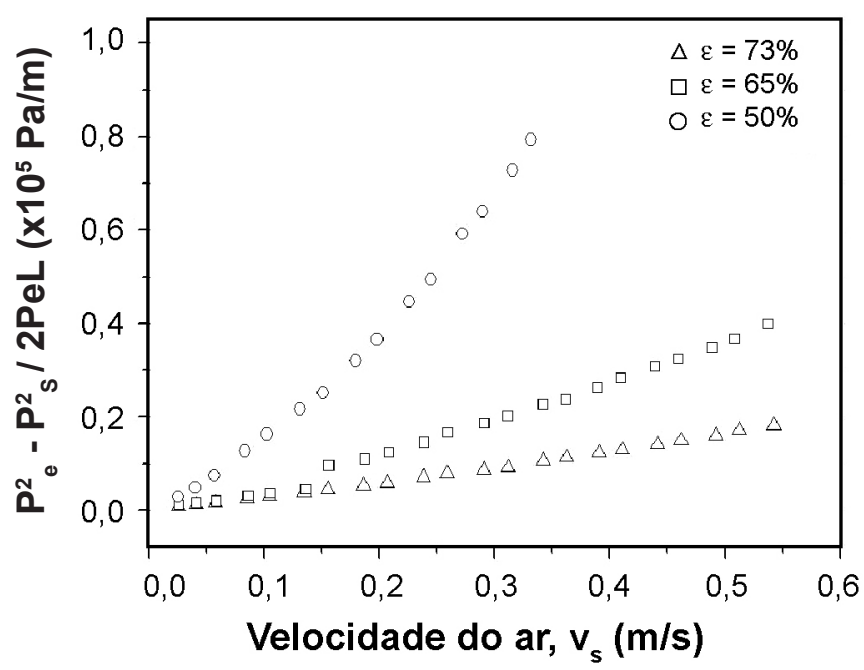

Figura 7: Curvas experimentais de queda de pressão em função da velocidade do ar para os filtros cerâmicos fibrosos.

[Figure 7: Pressure drop curves as a function of the air velocity for the fibrous ceramics.] 
constantes de permeabilidade tendem a aumentar com o aumento da porosidade, conforme esperado e já observado em literatura. A permeabilidade depende não somente da porosidade total, mas também do tamanho e número de poros na área de escoamento [11].

Como $k_{1}$ e $k_{2}$ também dependem da conexão entre os poros, que é definida por sua geometria, os filtros cerâmicos fibrosos apresentam alta permeabilidade considerando a anisotropia dos vazios formados por redes de fibras, comparadas com filtros $\mathrm{Al}_{2} \mathrm{O}_{3}-\mathrm{SiC}$ numa mesma faixa de porosidade (permeabilidade Darciana $\mathrm{k}_{1}$ tipicamente na faixa de $10^{-12}$ a $10^{-8} \mathrm{~m}^{2}$ ) $[11,13]$. Os filtros fibrosos silicoaluminosos aqui testados apresentam valores de resistência mecânica e permeabilidade na mesma faixa dos filtros comerciais para filtração de aerossóis, o que os qualifica, portanto, para diversas aplicações tecnológicas $[1,13,20]$.

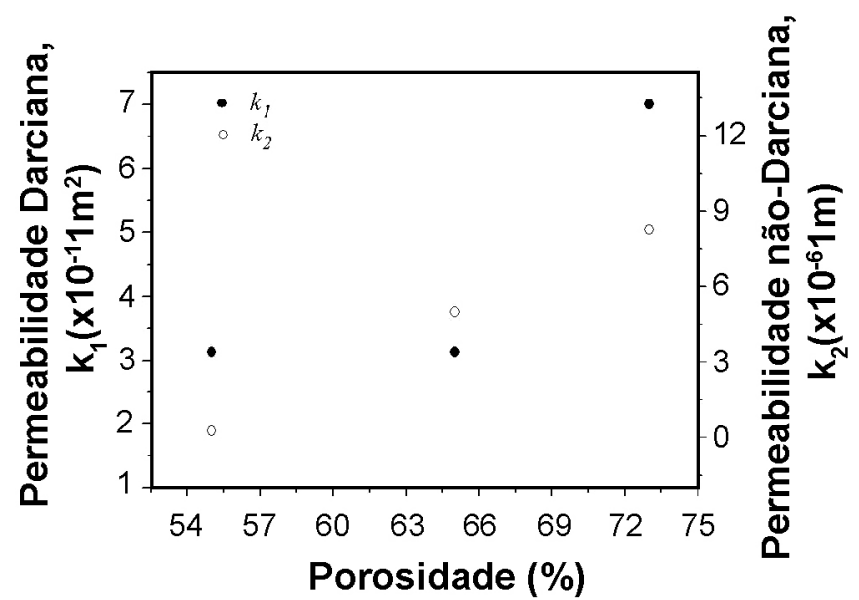

Figura 8: Permeabilidades darciana $\left(\mathrm{k}_{1}\right)$ e não-darciana $\left(\mathrm{k}_{2}\right)$ em função da porosidade dos filtros fibrosos.

[Figure 8: Darcian $\left(k_{1}\right)$ and non-Darcian $\left(k_{2}\right)$ permeability as a function of the porosity of the fibrous filters.]

\section{Filtração}

A Fig. 9 apresenta os resultados de eficiência de coleta de um filtro com $65 \%$ de porosidade, em função do diâmetro das partículas do aerossol para os tempos de filtração avaliados. Verifica-se que a eficiência de coleta foi bastante alta (eficiência global acima de $97 \%$ para todos os tempos de filtração) o que caracteriza o filtro cerâmico fibroso como uma alternativa muito promissora para limpeza de gases. Na verdade, para efeitos ambientais, além da eficiência de coleta, a concentração de partículas na saída do filtro deve ser avaliada. A resolução CONAMA 382 de 2006 (CONAMA, 2006 estabelece que de modo médio, a concentração máxima de saída em processos de combustão deve ser da ordem de 200 a $280 \mathrm{mg} / \mathrm{m}^{3}$. Por outro lado, a taxa de emissão típica de um processo de combustão sem filtro é da ordem de 3500 a $6000 \mathrm{mg} / \mathrm{m}^{3}$ (Coelho, 1999). Assim, é necessária uma eficiência global da ordem de 97\% para atender ao limite de emissão. Pelos dados obtidos neste trabalho, observa-se que todos os filtros apresentaram eficiência acima desse valor, confirmando sua aplicabilidade para a redução da emissão de particulados para a atmosfera em processos de combustão em fontes fixas.

Pode-se observar que a eficiência de coleta foi crescente até o diâmetro de partícula de $4,0 \mu \mathrm{m}$, mostrando a predominância do mecanismo de coleta inercial. Entretanto, para diâmetros de partícula maiores que $4,0 \mu \mathrm{m}$, observa-se um decréscimo na eficiência de coleta, talvez em decorrência do rebatimento das partículas após o contato com o coletor. Observa-se também um aumento na eficiência de coleta ao longo do tempo de filtração. Este comportamento é característico de filtros tipo barreira, pois após a retenção as partículas coletadas contribuem para captura de novas partículas. Os resultados obtidos para ensaios de filtração estão em ordem de grandeza esperada, para aplicações em filtração de aerossóis [2-8].

\section{CONCLUSÕES}

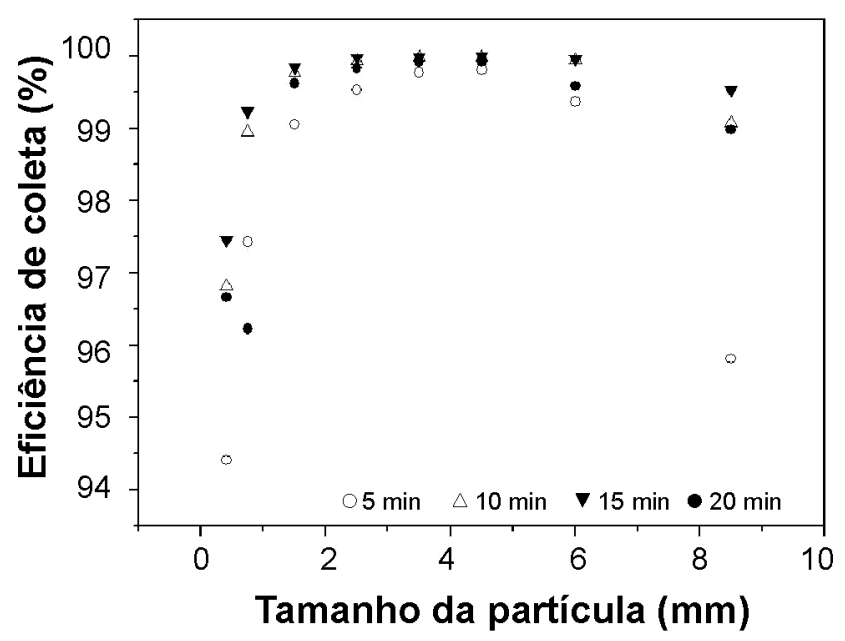

Figura 9: Eficiência de coleta em função do diâmetro de partícula para o filtro cerâmico fibroso com porosidade de $65 \%$.

[Figure 9: Collection efficiency as a function of the particle diameter of a fibrous filter with porosity of 65\%.]

Filtros fibrosos compostos de mulita com porosidade interconectada foram obtidos pelo processo de prensagem partindo de fibras aluminossilicosas comerciais. Com base nos resultados de permeabilidade e eficiência de coleta podese concluir que os filtros cerâmicos fibrosos apresentam características adequadas para filtração de gases e aerossóis. A resistência mecânica dos filtros mostrou-se apropriada para seu manuseio e sua utilização em condições reais de operação. Os resultados obtidos, tanto fluidodinâmicos quanto mecânicos, sugerem grande potencial dos materiais fibrosos para outras aplicações industriais, tais como combustão de diesel em veículos automotivos, craqueamento catalítico e refino de metais.

\section{AGRADECIMENTOS}

Ao CNPq, pelo suporte financeiro, e à Tecnotermo, por ter gentilmente cedido as mantas refratárias. 


\section{REFERÊNCIAS}

[1] P. Pastila, V. Helanti, A. P. Nikkilä, T. Mäntylä, J. Eur. Ceram. Soc. 21 (2001) 1261.

[2] N. L. Freitas, J. A. S. Gonçalves, M. D. M. Innocentini, J. R. Coury, J. Hazard. Mater. B136 (2006) 747.

[3] M. J. Metteson, Filtration: principles and practices, $2^{\text {nd }}$ Ed., New York, EUA (1987) 736.

[4] B. A. Latella, L. Henkel, E. G. Mehrtens, J. Mater. Sci. 41 (2006) 423.

[5] J. A. Fernado, D. D. L. Chung, J. Porous Mat. 9 (2002) 211.

[6] J. Steffens, J. R. Coury, Sep. Purif. Technol. (2007) em impressão.

[7] M. D. M. Innocentini, V. C. Pandolfelli, J. Am. Ceram. Soc. 84 (2001) 941.

[8] N. L. Freitas, M. G. Maneiro, J. R. Coury, Cerâmica 50, 316 (2004) 355.

[9] V. R. Salvini, M. D. M. Innocentini, V. C. Pandolfelli, Cerâmica 48, 307 (2002) 121.

[10] V. R. Salvini, M. D. M. Innocentini, V. C. Pandolfelli, Cerâmica 46, 298 (2000) 97.
[11] M. D. M. Innocentini, P. Sepulveda, V. R. Salvini, V. C. Pandolfelli, J. Am. Ceram. Soc. 81 (1998) 3349.

[12] M. D. M. Innocentini, V. R. Salvini, V. C. Pandolfelli, J. R. Coury, J. Am. Ceram. Soc. 82 (1999) 1945.

[13] V. R. Salvini, A. M. Pupim, M. D. M. Innocentini, V. C. Pandolfelli, Cerâmica 47, 301 (2001) 13.

[14] M. D. M. Innocentini, P. Sepulveda, F. Ortega, Permeability, in M. Scheffler, P. Colombo (Eds.), Cellular Ceramics: Structure, Manufacturing, Properties Applications (2005) 313-340.

[15] D. J. Dyson, M. A. Butler, R. J. Hughes, R. Fisher, G. W. Hicks, Ann. Occup. Hyg. 41 (1997) 561.

[16] L. J. Gibson, M. F. Ashby, Cellular solids, structure and properties, $2^{\text {nd }}$ Ed., Cambridge University Press, Cambridge, UK (1997) 175.

[17] A. S. Wagh, R. B. Poeppel, J. P. Singh, J. Mater. Sci. 26 (1991) 3862.

[18] L. J. Gibson, J. Biomech. 38 (2005) 377.

[19] F. A. C. Oliveira, S. Dias, M. F. Vaz, J. C. Fernandes, J. Eur. Ceram. Soc. 26 (2006) 179.

[20] E. A. Moreira, J. R. Coury, Braz. J. Chem. Eng. 21 (2004) 23.

(Rec. 18/04/2008, Ac. 18/12/2008) 